\title{
KILLING VECTOR FIELDS ON COMPLETE RIEMANNIAN MANIFOLDS
}

\author{
SHINSUKE YOROZU
}

\begin{abstract}
We discuss Killing vector fields with finite global norms on complete Riemannian manifolds whose Ricci curvatures are nonpositive or negative.
\end{abstract}

1. It is well known that if a compact Riemannian manifold has nonpositive Ricci curvature then every Killing vector field is a parallel vector field (cf. [3]). In this note, we discuss Killing vector fields with finite global norms on complete Riemannian manifolds. One of our results is that if $M$ is a complete Riemannian manifold with nonpositive Ricci curvature then every Killing vector field on $M$ with finite global norm is a parallel vector field. This is a generalization of the above well-known result. We also discuss the volume of a complete noncompact Riemannian manifold with nonpositive Ricci curvature. Our ideas are based on those of the papers of A. Andreotti and E. Vesentini [1] and, especially, H. Kitahara [2].

We shall be in the $C^{\infty}$-category. The manifolds considered are connected and orientable. The indices $h, i, j, k, \ldots$ run over the range $\{1,2, \ldots, n\}$ and the Einstein summation convention will be used.

2. Let $M$ be an $n$-dimensional complete Riemannian manifold and $g$ (resp. $\nabla$ ) its Riemannian metric tensor field (resp. its Levi-Civita connection). Let $\{U$ : $\left.\left(x^{1}, \ldots, x^{n}\right)\right\}$ denote a local coordinate system on $M . g_{i j}$ denotes the components of $g$ and $\left(g^{i j}\right)$ denotes the inverse matrix of the matrix $\left(g_{i j}\right)$. We set $\nabla_{i}=\nabla_{\partial / \partial x^{i}}$ and $\nabla^{i}=g^{i j} \nabla_{j}$

Let $\Lambda^{s}(M)$ be the space of all $s$-forms on $M$ and $\Lambda_{0}^{s}(M)$ the subspace of $\Lambda^{s}(M)$ composed of forms with compact supports. $\eta \in \Lambda^{s}(M)$ may be expressed locally as

$$
\eta=(1 / s !) \eta_{i_{1}} \cdots i_{s} d x^{i_{1}} \wedge \cdots \wedge d x^{i_{s}} .
$$

Let $\langle$,$\rangle denote the local scalar product; the global scalar product \langle\langle\rangle$,$\rangle is$ defined by

$$
\langle\langle\xi, \eta\rangle\rangle=\int_{M}\langle\xi, \eta\rangle * 1=\int_{M} \xi \wedge^{*} \eta
$$

for any $\xi, \eta \in \Lambda_{0}^{s}(M)$, where * denotes the star operator (cf. [4]). Let $L_{2}^{s}(M)$ be the completion of $\Lambda_{0}^{s}(M)$ with respect to the scalar product $\langle\langle\rangle\rangle . d:, \Lambda^{s}(M) \rightarrow$ $\Lambda^{s+1}(M)$ denotes the exterior derivative and $\delta: \Lambda^{s}(M) \rightarrow \Lambda^{s-1}(M)$ is defined by

Received by the editors February 12, 1981 and, in revised form, March 16, 1981.

1980 Mathematics Subject Classification. Primary 53C20.

Key words and phrases. Complete Riemannian manifold, Ricci curvature, Killing vector field. 
$\delta=(-1)^{s+n+1} * d *$. Then we have $\langle\langle d \xi, \eta\rangle\rangle=\langle\langle\xi, \delta \eta\rangle\rangle$ for any $\xi \in \Lambda_{0}^{s}(M)$, $\eta \in \Lambda_{0}^{s+1}(M)$. The Laplacian operator $\Delta$ acting on $\Lambda^{*}(M)=\Sigma_{s} \Lambda^{s}(M)$ is defined by $\Delta=d \delta+\delta d$.

For $\xi \in \Lambda^{1}(M)$, we have

$$
\begin{gathered}
(d \xi)_{i j}=\nabla_{i} \xi_{j}-\nabla_{j} \xi_{i}, \\
\delta \xi=-\nabla^{i} \xi_{i}, \\
(\Delta \xi)_{i}=-\nabla^{j} \nabla_{j} \xi_{i}-R_{\cdot i}^{h^{\circ} \xi_{h},}
\end{gathered}
$$

where $R_{\cdot i}^{h^{\cdot}}$ denotes the components of the Ricci tensor field of $\nabla$ (cf. [4]).

Hereafter, we identify the vector fields and its dual 1 -forms with respect to $g$ and they are represented by the same letters. For a vector field $\xi=\xi^{i} \partial / \partial x^{i}$, we have its dual 1-form $\xi=\xi_{j} d x^{j}=g_{i j} \xi^{i} d x^{j}$.

A vector field $\xi$ on $M$ is called a Killing vector field if $\mathcal{L}_{\xi} g=0$ where $\mathscr{L}$ denotes the Lie derivative operator. A Killing vector field $\xi$ satisfies the following:

$$
\nabla_{i} \xi_{j}+\nabla_{j} \xi_{i}=0
$$

and, from this, we have

$$
\nabla^{i} \xi_{i}=0 .
$$

A Killing vector field on $M$ is called "with finite global norm" if its dual 1-form with respect to $g$ belongs in $L_{2}^{1}(M) \cap \Lambda^{1}(M)$.

3. Let $o$ be a point of $M$ and fix it. For each point $p \in M$, we denote by $\rho(p)$ the geodesic distance from $o$ to $p$. Let $B(\alpha)=\{p \in M \mid \rho(p)<\alpha\}$ for $\alpha>0$. We choose a $C^{\infty}$-function $\mu$ on $\mathbf{R}$ (the reals) satisfying

(i) $0 \leqslant \mu(t) \leqslant 1$ on $\mathbf{R}$,

(ii) $\mu(t)=1$ for $t \leqslant 1$,

(iii) $\mu(t)=0$ for $t \geqslant 2$, and we set

$$
w_{\alpha}(p)=\mu(\rho(p) / \alpha)
$$

for $\alpha=1,2,3, \ldots$ Then we have

LEMMA 1 (cf. [1]). There exists a positive number $A$, depending only on $\mu$, such that

$$
\begin{gathered}
\left\|d w_{\alpha} \wedge \xi\right\|_{B(2 \alpha)}^{2} \leqslant\left(n A / \alpha^{2}\right)\|\xi\|_{B(2 \alpha)}^{2}, \\
\left\|d w_{\alpha} \wedge^{*} \xi\right\|_{B(2 \alpha)}^{2} \leqslant\left(n A / \alpha^{2}\right)\|\xi\|_{B(2 \alpha)}^{2}
\end{gathered}
$$

for any $\xi \in \Lambda^{s}(M)$, where

$$
\|\xi\|_{B(2 \alpha)}^{2}=\langle\langle\xi, \xi\rangle\rangle_{B(2 \alpha)}=\int_{B(2 \alpha)}\langle\xi, \xi\rangle * 1 .
$$

We remark that, for $\xi \in L_{2}^{s}(M) \cap \Lambda^{s}(M), w_{\alpha} \xi$ belongs in $\Lambda_{0}^{s}(M)$ and $w_{\alpha} \xi \rightarrow \xi$ $(\alpha \rightarrow \infty)$ in the strong sense. 
For any $\xi \in L_{2}^{1}(M) \cap \Lambda^{1}(M)$, we have

$$
\begin{gathered}
d \xi_{\alpha}=w_{\alpha}^{2} d \xi+2 w_{\alpha} d w_{\alpha} \wedge \xi, \\
\delta \xi_{\alpha}=w_{\alpha}^{2} \delta \xi-{ }^{*}\left(2 w_{\alpha} d w_{\alpha} \wedge^{*} \xi\right),
\end{gathered}
$$

where $\xi_{\alpha}=w_{\alpha}^{2} \xi$.

LEMMA 2 (cf. [2]). For any $\xi \in L_{2}^{1}(M) \cap \Lambda^{1}(M)$,

$\left\langle\left\langle 2 w_{\alpha} d w_{\alpha} \wedge \xi, \nabla \xi\right\rangle\right\rangle_{B(2 \alpha)}+\left\langle\left\langle w_{\alpha} \nabla^{2} \xi, w_{\alpha} \xi\right\rangle\right\rangle_{B(2 \alpha)}+\left\langle\left\langle w_{\alpha} \nabla \xi, w_{\alpha} \nabla \xi\right\rangle\right\rangle_{B(2 \alpha)}=0$, where $\left(\nabla^{2} \xi\right)_{i}=\nabla^{j} \nabla_{j} \xi_{i}$ and $(\nabla \xi)_{i j}=\nabla_{i} \xi_{j}$.

Proof. For given $\xi$, we consider a 1-form $\eta$ defined by

$$
\eta=\frac{1}{2} d(\langle\xi, \xi\rangle)=\left(\nabla_{i} \xi_{j}\right) \xi^{j} d x^{i}
$$

Then, ${ }^{*}\left(w_{\alpha}^{2} \eta\right)$ being a $(n-1)$-form with compact support in $B(2 \alpha)$, we have

$$
\int_{M} d\left(*\left(w_{\alpha}^{2} \eta\right)\right)=0
$$

On the other hand, we have

$$
d\left({ }^{*}\left(w_{\alpha}^{2} \eta\right)\right)=-{ }^{*} \delta\left(w_{\alpha}^{2} \eta\right)
$$

Thus we have

$$
\int_{M}^{*} \delta\left(w_{\alpha}^{2} \eta\right)=0
$$

By (2.2) and (3.2), we have

$$
\delta\left(w_{\alpha}^{2} \eta\right)=-w_{\alpha}^{2}\left(\nabla^{i} \nabla_{i} \xi_{j}\right) \xi^{j}-w_{\alpha}^{2}\left(\nabla_{i} \xi_{j}\right)\left(\nabla^{i} \xi^{j}\right)-{ }^{*}\left(2 w_{\alpha} d w_{\alpha} \wedge^{*} \eta\right)
$$

Therefore we have the assertion.

Let $\xi$ be a Killing vector field on $M$ whose dual 1-form with respect to $g$ belongs in $L_{2}^{1}(M) \cap \Lambda^{1}(M)$. By the definition of $\Delta,(2.2)$ and (2.5), we have

$$
\left\langle\left\langle\Delta \xi, w_{\alpha}^{2} \xi\right\rangle\right\rangle_{B(2 \alpha)}-\left\langle\left\langle\delta d \xi, w_{\alpha}^{2} \xi\right\rangle\right\rangle_{B(2 \alpha)}=0
$$

From (2.3), we have

$$
\left\langle\left\langle\Delta \xi, w_{\alpha}^{2} \xi\right\rangle\right\rangle_{B(2 \alpha)}=-\left\langle\left\langle w_{\alpha} \nabla^{2} \xi, w_{\alpha} \xi\right\rangle\right\rangle_{B(2 \alpha)}+\left\langle\left\langle w_{\alpha} \Re \xi, w_{\alpha} \xi\right\rangle\right\rangle_{B(2 \alpha)},
$$

where $\Re$ denotes the Ricci transformation on 1-forms defined by $(\Re \xi)_{i}=-R_{\cdot i}^{h^{\bullet}} \xi_{h}$. On the other hand, by (3.1), we have

$$
\left\langle\left\langle\delta d \xi, w_{\alpha}^{2} \xi\right\rangle\right\rangle_{B(2 \alpha)}=\left\langle\left\langle w_{\alpha} d \xi, w_{\alpha} d \xi\right\rangle\right\rangle_{B(2 \alpha)}+\left\langle\left\langle d \xi, 2 w_{\alpha} d w_{\alpha} \wedge \xi\right\rangle\right\rangle_{B(2 \alpha)}
$$

By (2.4),

$$
\begin{aligned}
\langle d \xi, d \xi\rangle & =(1 / 2 !)\left\{2\left(\nabla_{i} \xi_{k}\right)\left(\nabla^{i} \xi^{k}\right)-2\left(\nabla_{i} \xi_{k}\right)\left(\nabla^{k} \xi^{i}\right)\right\} \\
& =(1 / 2 !)\left\{2\left(\nabla_{i} \xi_{k}\right)\left(\nabla^{i} \xi^{k}\right)+2\left(\nabla_{i} \xi_{k}\right)\left(\nabla^{i} \xi^{k}\right)\right\} \\
& =(1 / 2 !) 4\left(\nabla_{i} \xi_{k}\right)\left(\nabla^{i} \xi^{k}\right) \\
& =4\langle\nabla \xi, \nabla \xi\rangle
\end{aligned}
$$

and we have

$$
\left\|w_{\alpha} d \xi\right\|_{B(2 \alpha)}^{2}=4\left\|w_{\alpha} \nabla \xi\right\|_{B(2 \alpha)}^{2}
$$


By the Schwarz inequality, Lemma 1 and the above fact, we have

$$
\begin{aligned}
\left|\left\langle\left\langle d \xi, 2 w_{\alpha} d w_{\alpha} \wedge \xi\right\rangle\right\rangle_{B(2 \alpha)}\right| & \leqslant\left\|w_{\alpha} d \xi\right\|_{B(2 \alpha)}\left\|2 d w_{\alpha} \wedge \xi\right\|_{B(2 \alpha)} \\
& \leqslant \frac{1}{2}\left(\left\|w_{\alpha} d \xi\right\|_{B(2 \alpha)}^{2}+4\left\|d w_{\alpha} \wedge \xi\right\|_{B(2 \alpha)}^{2}\right) \\
& \leqslant \frac{1}{2}\left(4\left\|w_{\alpha} \nabla \xi\right\|_{B(2 \alpha)}^{2}+\left(4 n A / \alpha^{2}\right)\|\xi\|_{B(2 \alpha)}^{2}\right) .
\end{aligned}
$$

Thus we have, from (3.3),

$$
\begin{aligned}
\left\langle\left\langle w_{\alpha} \Re \xi, w_{\alpha} \xi\right\rangle\right\rangle_{B(2 \alpha)}= & \left\langle\left\langle w_{\alpha} \nabla^{2} \xi, w_{\alpha} \xi\right\rangle\right\rangle_{B(2 \alpha)}+\left\langle\left\langle w_{\alpha} d \xi, w_{\alpha} d \xi\right\rangle\right\rangle_{B(2 \alpha)} \\
& +\left\langle\left\langle d \xi, 2 w_{\alpha} d w_{\alpha} \wedge \xi\right\rangle\right\rangle_{B(2 \alpha)} \\
\geqslant & \left\langle\left\langle w_{\alpha} \nabla^{2} \xi, w_{\alpha} \xi\right\rangle\right\rangle_{B(2 \alpha)}+4\left\|w_{\alpha} \nabla \xi\right\|_{B(2 \alpha)}^{2} \\
& -\frac{1}{2}\left(4\left\|w_{\alpha} \nabla \xi\right\|_{B(2 \alpha)}^{2}+\left(4 n A / \alpha^{2}\right)\|\xi\|_{B(2 \alpha)}^{2}\right)
\end{aligned}
$$

(by Lemma 2)

$$
\begin{aligned}
= & -\left\langle\left\langle w_{\alpha} \nabla \xi, w_{\alpha} \nabla \xi\right\rangle\right\rangle_{B(2 \alpha)}-\left\langle\left\langle 2 w_{\alpha} d w_{\alpha} \wedge \xi, \nabla \xi\right\rangle\right\rangle_{B(2 \alpha)} \\
& +4\left\|w_{\alpha} \nabla \xi\right\|_{B(2 \alpha)}^{2}-\frac{1}{2}\left(4\left\|w_{\alpha} \nabla \xi\right\|_{B(2 \alpha)}^{2}+\left(4 n A / \alpha^{2}\right)\|\xi\|_{B(2 \alpha)}^{2}\right)
\end{aligned}
$$

(by the Schwarz inequality and Lemma 1)

$$
\begin{aligned}
\geqslant & -\left\|w_{\alpha} \nabla \xi\right\|_{B(2 \alpha)}^{2}-\frac{1}{2}\left(\left\|w_{\alpha} \nabla \xi\right\|_{B(2 \alpha)}^{2}+\left(4 n A / \alpha^{2}\right)\|\xi\|_{B(2 \alpha)}^{2}\right) \\
& +4\left\|w_{\alpha} \nabla \xi\right\|_{B(2 \alpha)}^{2}-\frac{1}{2}\left(4\left\|w_{\alpha} \nabla \xi\right\|_{B(2 \alpha)}^{2}+\left(4 n A / \alpha^{2}\right)\|\xi\|_{B(2 \alpha)}^{2}\right) .
\end{aligned}
$$

Therefore we have

$$
\left\langle\left\langle w_{\alpha} \Re \xi, w_{\alpha} \xi\right\rangle\right\rangle_{B(2 \alpha)} \geqslant \frac{1}{2}\left\|w_{\alpha} \nabla \xi\right\|_{B(2 \alpha)}^{2}-\left(4 n A / \alpha^{2}\right)\|\xi\|_{B(2 \alpha)}^{2} .
$$

Letting $\alpha \rightarrow \infty$, we have

LEMMA 3. Let $\xi$ be a Killing vector field on $M$ with finite global norm. If $\lim \sup _{\alpha \rightarrow \infty}\left\langle\left\langle w_{\alpha} \mathscr{R} \xi, w_{\alpha} \xi\right\rangle\right\rangle_{B(2 \alpha)}<\infty$, then

$$
\limsup _{\alpha \rightarrow \infty}\left\langle\left\langle w_{\alpha} \Re \xi, w_{\alpha} \xi\right\rangle\right\rangle_{B(2 \alpha)} \geqslant \frac{1}{2}\|\nabla \xi\|^{2} .
$$

THEOREM 1. If $M$ is a complete Riemannian manifold with nonpositive Ricci curvature, then every Killing vector field on $M$ with finite global norm is a parallel vector field.

ProOF. By the nonpositivity of Ricci curvature, we have

$$
\limsup _{\alpha \rightarrow \infty}\left\langle\left\langle w_{\alpha} \Re \xi, w_{\alpha} \xi\right\rangle\right\rangle_{B(2 \alpha)} \leqslant 0
$$

for any Killing vector field $\xi$ on $M$ with finite global norm. From Lemma 3, we have $\nabla \xi=0$.

Since the length of a parallel vector field is constant, we have

COROLlARY 1. Let $M$ be a complete noncompact Riemannian manifold with nonpositive Ricci curvature. If there exists a nontrivial Killing vector field on $M$ with finite global norm, then the volume of $M$ is finite.

The following example illustrates the role of the hypothesis that $M$ has nonpositive Ricci curvature in the above results. 
EXAMPLE 1. We take four real numbers $a_{1}, a_{2}, a_{3}$ and $a_{4}$ such that $0<a_{1}<a_{2}<$ $a_{3}<a_{4}<1$ and fix them. We consider two $C^{\infty}$-functions $h_{1}, h_{2}:(0, \infty) \rightarrow \mathbf{R}$ satisfying $0 \leqslant h_{i}(r) \leqslant 1(i=1,2)$ for $0<r$ and

$$
\begin{array}{lll}
h_{1}(r)=1, & h_{2}(r)=0 & \text { for } 0<r \leqslant a_{2}, \\
h_{1}(r)=0, & h_{2}(r)=1 & \text { for } a_{3} \leqslant r .
\end{array}
$$

We define functions $f_{i}, g_{i}(i=1,2)$ as follows; $f_{1}(r)=h_{1}(r) r^{-2}(\log r)^{-2}, g_{1}(r)=$ $h_{1}(r)(\log r)^{-2}$ for $0<r<a_{4}$ and $f_{2}(r)=h_{2}(r), g_{2}(r)=h_{2}(r) r^{-4 / 3}$ for $a_{1}<r$. Then we set

$$
\begin{array}{llll}
F_{1}(r)=f_{1}(r) & \left(0<r \leqslant a_{3}\right), & =0 & \left(a_{3}<r\right), \\
F_{2}(r)=0 & \left(0<r<a_{2}\right), & =f_{2}(r) & \left(a_{2}<r\right), \\
G_{1}(r)=g_{1}(r) & \left(0<r \leqslant a_{3}\right), & =0 & \left(a_{3}<r\right), \\
G_{2}(r)=0 & \left(0<r<a_{2}\right), & =g_{2}(r) & \left(a_{2}<r\right),
\end{array}
$$

and

$$
F(r)=F_{1}(r)+F_{2}(r), \quad G(r)=G_{1}(r)+G_{2}(r) \text { for } 0<r .
$$

The functions $F$ and $G$ are $C^{\infty}$ and $F(r), G(r)>0$ for $0<r$.

Let $M=R^{2}-\{(0,0)\}=\{(r, \theta) \mid 0<r, 0 \leqslant \theta<2 \pi\}$ and $d s^{2}=F(r)(d r)^{2}+$ $G(r)(d \theta)^{2}$. Then $\left(M, d s^{2}\right)$ is a complete Riemannian manifold. A vector field $\xi=\partial / \partial \theta$ on $M$ is a Killing vector field with respect to the Riemannian metric $d s^{2}$. Since $\int_{0}^{a_{2}} r^{-1}(\log r)^{-N} d r<\infty(N=2,3, \ldots), \int_{a_{3}}^{\infty} r^{-2 / 3} d r=\infty$ and $\int_{a_{3}}^{\infty} r^{-L} d r<$ $\infty(1<L)$, we have that the volume of $M$ is infinite, $\|\xi\|$ is finite and $0<$ $\langle\langle\Re \xi, \xi\rangle\rangle\langle\infty$.

REMARK TO COROllary 1. Every complete noncompact Riemannian manifold with nonnegative Ricci curvature has infinite volume (cf. S.-T. Yau [Indiana Univ. Math. J. 25 (1976), 659-670] and E. Calabi [Notices Amer. Math. Soc. 22 (1975), A205]).

THEOREM 2. If $M$ is a complete Riemannian manifold with negative Ricci curvature, then there is no nontrivial Killing vector field on $M$ with finite global norm.

Proof. Let $\xi$ be a Killing vector field on $M$ with finite global norm. By the negativity of Ricci curvature, we have $\left\langle\left\langle w_{\alpha} \Re \xi, w_{\alpha} \xi\right\rangle\right\rangle_{B(2 \alpha)} \leqslant 0$ for every $\alpha$. From Lemma 3,

$$
0 \geqslant \limsup _{\alpha \rightarrow \infty}\left\langle\left\langle w_{\alpha} \Re \xi, w_{\alpha} \xi\right\rangle\right\rangle_{B(2 \alpha)} \geqslant \frac{1}{2}\|\nabla \xi\|^{2} \geqslant 0 .
$$

Thus we have $\left\langle\left\langle w_{\alpha} \Re \xi, w_{\alpha} \xi\right\rangle\right\rangle_{B(2 \alpha)}=0$ for every $\alpha$. By the negativity of Ricci curvature, $\xi=0$.

REMARK. There is a similar discussion for holomorphic vector fields on complete Kähler manifolds with finite global norms [5].

Acknowledgements. The author expresses his hearty thanks to Professor $\mathbf{H}$. Kitahara for his valuable advice and to the referee for his kind comments. 


\section{REFERENCES}

1. A. Andreotti and E. Vesentini, Carleman estimates for the Laplace-Beltrami equation on complex manifolds, Inst. Hautes Etudes Sci. Publ. Math. 25 (1965), 313-362.

2. H. Kitahara, Non-existence of non-trivial $\square$ "-harmonic 1-forms on a complete foliated riemannian manifold, Trans. Amer. Math. Soc. 262 (1980), 429-435.

3. S. Kobayashi and K. Nomizu, Foundations of differential geometry, Vol. I, Interscience, New York, 1963.

4. G. de Rham, Variétés différentiables, Hermann, Paris, 1955.

5. S. Yorozu, Holomorphic vector fields on complete Kähler manifolds, Ann. Sci. Kanazawa Univ. 17 (1980), 17-21.

Department of Mathematics, College of liberal Arts, Kanazawa University, Kanazawa 920, JAPAN 